
of $y$.

This approximates closely to the exponential distribution for a wide range

The corresponding double-sided distribution is given by

$$
P(y)=\frac{1}{2 \sqrt{6 \pi \sigma_{y}}}\left[-E_{i}\left(-\frac{y^{2}}{6 \sigma^{2}}\right)\right]
$$

and in this case the difference between the time and statistical averages of the variance does not arise. This distribution also approximates closely to the exponential distribution over a range of values of $y$.

\title{
A Survey of the use of Astronomical Methods at Sea
}

\author{
D. McG. Bissett and J. J. Evans
}

THIs investigation of methods used in Astronomical Navigation has been carried out by means of questionnaires completed by senior students at a few of our Navigation Schools. The data has been classified into fairly broad categories since some of it has been rather difficult to assess, due to incomplete information on such details as trading areas, which watch was being kept, and the actual time spent at sea during the year.

The graphical analysis shows the frequency of usage for each method expressed as a percentage of the total number of observers questioned.

With regard to Solar observations the information obtained is generally as expected. The position line by Marcq. St. Hilaire is favoured a little more than Longitude by Chronometer.

(a) 42 per cent use only M. St. H. ; 27 per cent use only the longitude method; 27 per cent vary between these two methods, and only 4 per cent make use of short method tables, and

(b) of those who take ex-meridians, 9 I per cent make use of ex-meridian tables, the remainder working the calculations in full.

The Moon is noteworthy, if only for a conspicuous lack of observations, even of time azimuths. It would seem reasonable to expect that at some time of the day or night the Moon would be suitably disposed for observation, but there remains a peculiar reluctance to use it.

Considering the comparative infrequency of occasions when it is possible to observe planets, it is a little surprising to find them used so widely. Perhaps it is a matter of professional pride to the observer when he manages to locate, observe, and cross Venus with a Sun sight.

Despite a growing emphasis on the use of short method tables in the computation of zenith distance from star sights, our investigation revealed that of those regularly using stars for position fixing, 73 per cent work the calculation in full [ 5 per cent by longitude methods; $5^{8}$ per cent by $M$. St. H.] and only 27 per cent by short methods. 


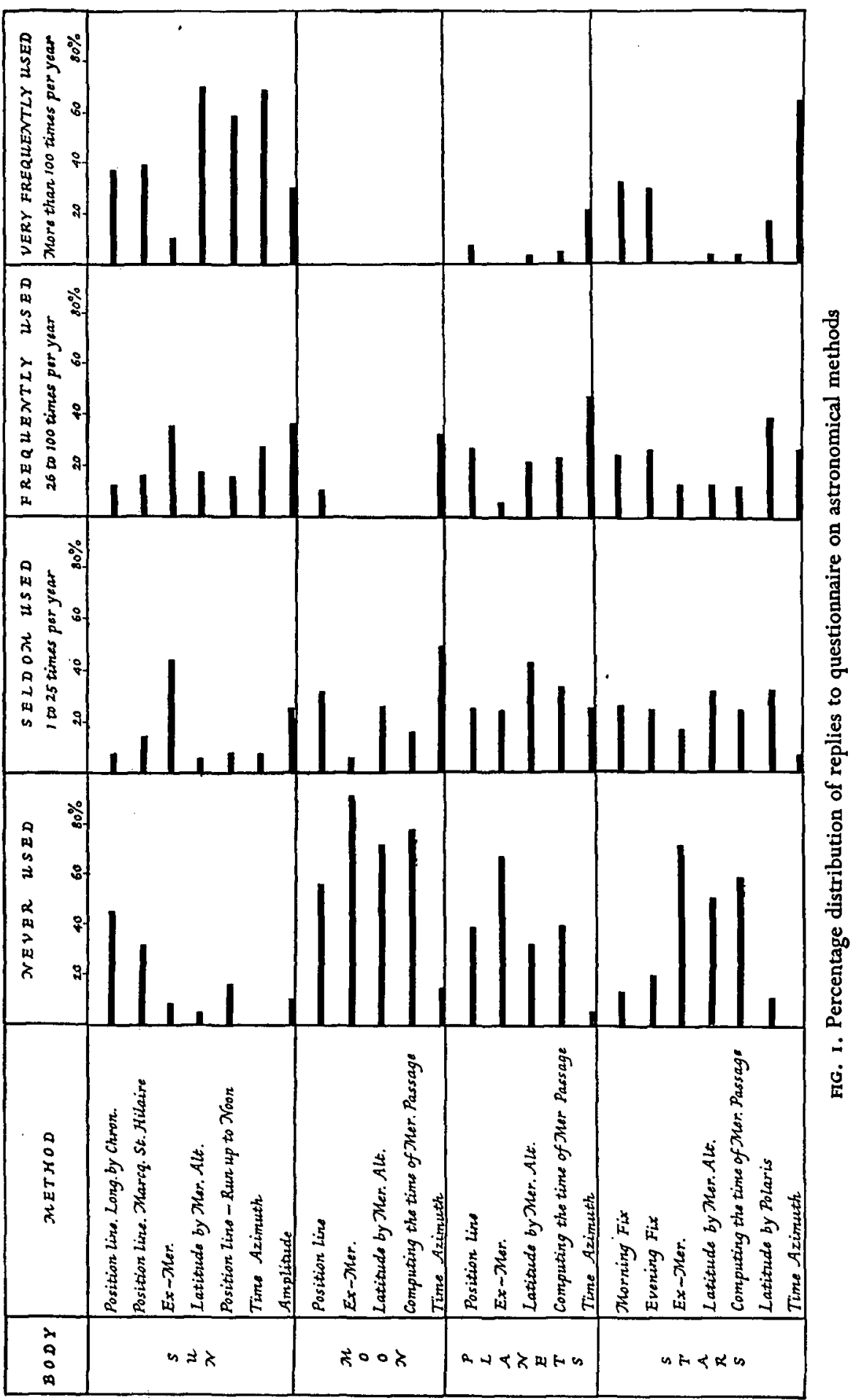


Many navigators made reference to position finding by crossing an astronomical position line with a position line obtained by other well-known methods such as Loran, Decca, Consol, radar, W/T bearing, and visual terrestrial bearings.

It is interesting to record that one observer claimed the occasional use of Equal Altitudes for obtaining a longitude at noon, and another listed Sumner's Method for a position line. Only three observers mentioned drawing position circles when the Sun was near the zenith.

\section{The Vinland Map}

THE discovery of the Vinland Map and the recent publication of its description* has been attended with considerable publicity on both sides of the Atlantic, in publications ranging from learned journals to glossies. Briefly, the map, provisionally dated about $145^{\circ}$, was discovered by an antiquarian bookseller of New Haven, Connecticut, in circumstances which so far have precluded a proper examination of its history. It has, as a recent reviewer put it, 'no ancestors, no close relations and no descendants'. Its importance to the student of the history of navigation and discovery, however, is that (assuming it is authentic) it is by far the earliest surviving example of a map portraying any part of the Americas, and it ostensibly provides the first evidence for an early school of Norse cartography, for Vinland can clearly be identified with that part of the American continent settled by the Norsemen in the latter part of the tenth century.

Professor E. G. R. Taylor, one of the leading authorities on ancient cartography and on the history of exploration and navigation, was shown, in confidence, a facsimile of the map some years before its publication and expressed misgivings about its authenticity. Her suspicions were first aroused in 1963 when she laid a tracing she happened to be making of the coasts of Newfoundland and Labrador over the coast of Vinland as delineated on the Vinland facsimile. The coincidence of outline was sufficiently remarkable to lead her to a close examination of the whole map. Her enquiry, which relied almost entirely upon measurements, led her to the view that in all probability the map is some kind of counterfeit and that she can identify many of the sources used. The detailed proofs of this contention involve a great deal of illustration which the short time available between publication of the map and going to press with the Journal does not allow. However, the map has received such prominence that it was thought important to give an outline of Professor Taylor's contentions as soon as possible and to suggest that, until a more thorough investigation of the map's history is made, students of ancient cartography and the history of navigation might wish to keep an open mind on the question of the map's authenticity. The principal points Professor Taylor makes are as follows :

I. The eliptical boundary of the map fits remarkably closely the boundaries of one of the plates in The Elements of Map Projection by Deetz \& Adams, a

* The Vinland Map and the Tartar Relation, by R. A. Skelton, Thomas E. Marston and George D. Painter, for the Yale University Library, with a Foreword by Alexander $O$. Vietor; Yale University Press, New Haven and London; 1965. 